Background: Calcium pyrophosphate crystal deposition disease (CPPD) is associated with a high frequency of comorbidities and vascular calcification [1], but it is unknown whether these factors affect cardiovascular mortality.

Objectives: To study the structure of mortality in patients with CPPD and compare with the structure of mortality in the Russian Federation.

Methods: 217 patients with crystal-verified diagnosis of CPPD (McCarty criteria) were included in the prospective study, aged $\geq 18$ years, mean age $59.2 \pm$ 12.6 years, $82(38 \%)$ men and $134(62 \%)$ women. The median follow-up was 3.9 $[1.9 ; 7.2]$ years, patients were followed up for at least a year. The exclusion criteria are the presence of other rheumatic diseases with arthritis symptoms. The examination of patients included the history taking, assessment of anthropometric parameters, the presence of comorbidity and the following laboratory tests: determination of serum creatinine level (with calculation of glomerular filtration rate (eGFR) using the MDRD formula), total cholesterol (TC), C-reactive protein (CRP). Statistica 12.0 package was used for statistical data processing.

Results: A total of 217 patients were included. Arterial hypertension was detected in $115(53 \%)$ patients, coronary heart disease in 51 (24\%) patients, diabetes mellitus in $26(12 \%)$ patients, chronic renal failure in $17(8 \%)$ patients, hyperparathyroidism in $18(8 \%)$ patients, chronic heart failure in $22(10 \%)$ patients. 65 $(30 \%)$ patients had a family history for CVD, 31 (14\%) patients were smokers. $122(56 \%)$ patients had an increased level of total cholesterol> $5.0 \mathrm{mmol} / \mathrm{L}$ and $54(25 \%)$ patients - the level of CRP $>5 \mathrm{mg} / \mathrm{L}$

$23(11 \%)$ patients, $12(52 \%)$ men and $11(48 \%)$ women, died, the average age of the deceased being $62.7 \pm 9.2$ years. In $15(65 \%)$ cases out of 23 , death occurred due to cardiovascular complications, which is higher than the cardiovascular mortality rate in the Russian Federation (53\%). Among CVD, the distribution was as follows: acute myocardial infarction - $6(40 \%)$ patients,apoplectic attack - 5 (33) patients, thrombosis - 2 (13\%) patients, rhythm disturbances - 1 (7\%) patient and decompensation of chronic heart failure - 1 (7\%) patient.

Conclusion: CVD is the main cause of death in patients with CPPD and the total frequency of mortality from CVD exceeds the population one. Further research is needed, including studies of the risk factors for overall and cardiovascular mortality in patients with CPPD.

REFERENCES:

[1] Abhishek A, Doherty S, Maciewicz R, et al. Association between low corti-

cal bone mineral density, soft-tissue calcification, vascular calcification and chondrocalcinosis: a case-control study. Ann Rheum Dis. 2013;73(11):19972002. doi: 10.1136/annrheumdis2013-203400

Disclosure of Interests: Maxim Eliseev Speakers bureau: Berlin Chemie Menarini Group, Novartis International AG, EGIS, Aleksandra Novikova: None declared., Olga Sheliabina: None declared., Maria Chikina: None declared. DOI: 10.1136/annrheumdis-2021-eular.3142

\section{AB0641 EFFECT OF AGE ON PARATHYROID HORMONE RESPONSE TO VITAMIN D INSUFFICIENCY IN PRIMARY HYPERPARATHYROIDISM}

V. Deshani ${ }^{1}$, M. Khalid ${ }^{1}$, K. Jadoon ${ }^{1} .{ }^{1}$ The Royal Wolverhampton NHS Trust, Diabetes and Endocrinology, Wolverhampton, United Kingdom

Background: Primary hyperparathyroidism (PHPT) is a common endocrine condition, commonly seen with increasing age. In vast majority, it is diagnosed incidentally and causes no particular symptoms. Symptoms are usually related to acute hypercalcaemia or the complications of chronically elevated serum calcium level. Vitamin D deficiency is common among general population and in patients with PHPT. Studies in secondary hyperparathyroidism (SHPT) have shown that parathyroid hormone (PTH) response is affected by age, with those over 80 showing greater rise in PTH levels. We wanted to see if age has a similar impact on PTH response to vitamin D in those with PHPT.

Objectives: To evaluate the impact of age on PTH response to vitamin D insufficiency in those with PHPT.

Methods: Patients with primary hyperparathyroidism (PHPT), attending general endocrine clinic of a district general hospital, were divided into two groups based on age; less than $70(n=73)$ and 70 and above $(n=61)$. Each group was subdivided into vitamin D insufficient (VDI) and vitamin D sufficient (VDS) subgroups. We compared calcium and parathyroid hormone levels and forearm BMD (presented as T score) in VDI and VDS subgroups in the two age groups, at the time of diagnosis. Data were analyzed using unpaired t-test and presented as mean \pm SEM, using Graphpad Prism 9.0.1.

Results: There was significant difference in Vitamin D levels in VDI and VDS subgroups, in both age groups ( $<70$; mean vitamin D 27.98 vs. 68.44 , $p<0.0001 ; \geq 70$; mean vitamin D 34.44 vs. $75.74, p<0.0001)$. The two groups were significantly different in terms of age (mean age 58 vs. $76, p<0.0001$ ). Although there was no difference in calcium and forearm BMD in VDI and VDS, in both age groups, those under 70 showed a greater PTH response to vitamin D insufficiency (mean PTH 19.29 vs. 12.91 respectively, $p<0.001$ ). Conclusion: While in SHPT, those with increasing age show greater rise in $\mathrm{PTH}$ levels, our data show that in PHPT, younger patients show a greater PTH rise in response to vitamin $D$ insufficiency. Further work is needed to elucidate the underlying mechanisms.

\section{REFERENCES:}

[1] Wyskida et al., Parathyroid hormone response to different vitamin D levels in population-based old and very-old Polish cohorts, Experimental Gerontology, Volume 127, 2019, 110735, ISSN 0531-5565, https://doi.org/10.1016/j. exger.2019.110735

[2] Malik M Z, Latiwesh O B, Nouh F, et al. (August 15, 2020) Response of Parathyroid Hormone to Vitamin D Deficiency in Otherwise Healthy Individuals. Cureus 12(8): e9764. doi:10.7759/cureus.9764

Disclosure of Interests: None declared.

DOI: 10.1136/annrheumdis-2021-eular.3288

\section{AB0642 1 CLINICAL CHARACTERISTICS AND FACTORS ASSOCIATED WITH BONE EROSION IN GOUT PATIENTS WITH TOPHI}

W. Deng ${ }^{1}$, Y. Huang ${ }^{1}$, Y. Liu ${ }^{1}$, Z. Huang ${ }^{1}$, S. Chen ${ }^{1}$, Q. Huang ${ }^{1}$, T. W. LI ${ }^{1}$.

${ }^{1}$ Guangdong Second Provincial General Hospital, Department of

Rheumatology and Immunology, Guangzhou, China

Background: Bone erosion is a frequent complication of gout patients with tophi and can lead to joint damage, deformity and musculoskeletal disability. Few studies have focused on clinical characteristics and factors associated with bone erosion in gout patients with tophi.

Objectives: The aim of this study was to describe clinical characteristics of bone erosion in gout patients with tophi.

Methods: Bone erosion was detected by X-ray. Gout patients with tophi were divided into bone erosion group and non bone erosion group. The clinical characteristics were recorded. Comparison of clinical characteristics and risk factors for bone erosion were analyzed between two groups. Multivariate logistic regression analysis was conducted.

Results: A total of 171 gout patients with tophi were enrolled, 121 patients with bone erosion and 50 patients without bone erosion. Bone erosion group were older, with prolonged duration with gout and tophi, higher levels of serum creatinine, lower levels of glomerular filtration rate (GFR), C-reactive protein and BMI. In univariate regression analysis, age, gout duration, tophi duration, GFR were associated with bone erosion. In multivariable logistic regression analysis, tophi duration was independently associated with bone erosion.

Conclusion: Gout patients with bone erosion present different clinical characteristics compared with those without bone erosion. Tophi duration was strongly associated with bone erosion in patients with gout.

Table 1. Comparison of clinical characteristics between bone erosion patients and non bone erosion patients.

\begin{tabular}{llll}
\hline & Non Bone erosion & Bone erosion & $P$ Value \\
\hline $\mathrm{N}($ male) & $50(47)$ & $121(118)$ & 0.255 \\
Age $($ year) & $45.82 \pm 14.15$ & $53.74 \pm 14.88$ & 0.002 \\
$\mathrm{BMI}(\mathrm{kg} / \mathrm{m} 2)$ & $26.01 \pm 4.58$ & $24.18 \pm 4.72$ & 0.027 \\
$\mathrm{WBC}\left(10^{9} / \mathrm{mL}\right)$ & $9.73 \pm 3.40$ & $11.37 \pm 13.26$ & 0.404 \\
$\mathrm{PLT}\left(10^{9} / \mathrm{mL}\right)$ & $329.86 \pm 96.22$ & $328.31 \pm 124.02$ & 0.938 \\
$\mathrm{HGB}(\mathrm{g} / \mathrm{L})$ & $86.58 \pm 63.78$ & $102.75 \pm 51.16$ & 0.201 \\
$\mathrm{ALT}(\mathrm{U} / \mathrm{L})$ & $37.74 \pm 26.56$ & $34.26 \pm 35.26$ & 0.561 \\
$\mathrm{sUA}(\mathrm{umol} / \mathrm{L})$ & $540.16 \pm 121.79$ & $539.00 \pm 121.46$ & 0.962 \\
$\mathrm{sCr}($ umol/L) & $111.47 \pm 25.26$ & $135.77 \pm 52.43$ & $<0.001$ \\
$\mathrm{GFR}$ & $74.01 \pm 27.94$ & $56.68 \pm 22.84$ & 0.003 \\
& & & \\
(ml/min/1.73m & & & \\
ESR $(\mathrm{mm} / \mathrm{h})$ & & $53.08 \pm 36.70$ & 0.181 \\
$\mathrm{CRP}(\mathrm{mg} / \mathrm{L})$ & $61.78 \pm 37.32$ & $36.45 \pm 42.62$ & 0.014 \\
Gout duration (year) & $60.00 \pm 58.26$ & $12.63 \pm 7.59$ & 0.001 \\
Tophi duration(year) & $9.22 \pm 5.46$ & $6.64 \pm 4.81$ & 0.001 \\
Hypertension, $\mathrm{n}$ & $3.77 \pm 3.22$ & 52 & 0.277 \\
Diabetes, $\mathrm{n}$ & 17 & 12 & 0.143 \\
Smoking history, $\mathrm{n}$ & 9 & 55 & 0.513 \\
Drinking history, $\mathrm{n}$ & 20 & 37 & 0.737 \\
Ulceration, $\mathrm{n}$ & 14 & 35 & 0.228
\end{tabular}

Disclosure of Interests: None declared.

DOI: 10.1136/annrheumdis-2021-eular.3389

$\mathrm{AB} 0643$

IL-1 BLOCKAGE WITH ANAKINRA IN GOUT PATIENTS. SCOPING REVIEW OF THE PUBLISHED LITERATUR.

S. Jeria Navarro ${ }^{1}$, H. Park ${ }^{1}$, M. A. Pou ${ }^{2}$, E. Calvo-Aranda ${ }^{3}$, C. Diaz-Torne ${ }^{1}$

${ }^{1}$ Hospital de la Santa Creu i Sant Pau, Rheumatology, Barcelona, Spain; ${ }^{2} E A P$ Encants, ICS Atenció Primària, Barcelona, Spain; ${ }^{3}$ Hospital Universitario Infanta Leonor, Rheumatology, Madrid, Spain 